\title{
Psychiatrie und Psychotherapie: Der Fallkostendurchschnitt ist untauglich für die Wirtschaftlichkeitskontrolle
}

\author{
Stellungnahme zu den Rückzahlungsandrohungen der santésuisse an verschiedene Psychiater/innen in den Kantonen \\ Basel-Stadt und Basel-Landschaft / Abschaffung der Kontrahierungsverpflichtung
}

R. Balmer

Das Verfahren des Fallkostdurchschnittes ist ein untaugliches Mittel zur Kontrolle der Wirtschaftlichkeit. santésuisse wird aufgefordert, dieses aufzugeben und mit den betroffenen Verbänden andere Richtlinien zu erarbeiten. An Stelle der Aufhebung der Kontrahierungsverpflichtung soll eine umfassende psychiatrisch-psychotherapeutische Gesundheitspolitik entwickelt werden, die dem Umstand Rechnung trägt, dass psychisch kranke Menschen in diesem Lande noch immer unterversorgt sind. Diese Gesundheitspolitik muss unter anderem auf der Qualität der psychiatrisch-psychotherapeutischen Ausbildung aufbauen, wie dies die EFPP für ihre Mitglieder entwickelt hat [1].

1 EFPP-Argumentarium (Bezug: EFPP Schweiz, Spiegelbergstrasse 5, 4059 Basel).

2 Sandell, Blomberg, Lazar. Unterschiedliche Langzeitergebnisse von Psychoanalysen und Psychotherapie; Psyche 2001;55:277-310 Leuzinger-Bohleber, Stuhr, Rüger, Beutel. Langzeitwirkungen von Psychoanalysen und Psychotherapien - eine multiperspektivische, repräsentative Katamnesestudie. Psyche 2001;55:193-276.

Korrespondenz:

Dr. med. Rudolf Balmer

European Federation for

Psychoanalytic Psychotherapy

EFPP Schweiz

Spiegelbergstrasse 5

CH-4059 Basel
Als Organisation, welche in der Schweiz rund 400 psychoanalytisch ausgebildete Psychotherapeutinnen und -therapeuten - mit psychologischer und psychiatrischer Grundausbildung vertritt, erlauben wir uns, zu den eingangs gestellten Themen Stellung zu nehmen.

Regelmässig in den Herbstmonaten erhalten in den verschiedensten Kantonen Psychiaterinnen und Psychiater von der santésuisse, dem Dachverband der Krankenversicherer, Briefe, in denen sie auf überdurchschnittliche Fallkosten hingewiesen werden (Kostenaufwand pro Patient in einem Jahr). Diese Mitteilungen sind mit der Aufforderung verbunden, die Behandlungspraxis umgehend zu ändern, unter Androhung von Rückzahlungsforderungen im Wiederholungsfalle. Dieses Jahr hat in den Kantonen BS und BL eine Vielzahl von Psychiaterinnen und Psychiatern solche Briefe erhalten. Die santésuisse meint, mit diesem Vorgehen der gesetzlichen Aufforderung nach Kontrolle der Wirtschaftlichkeit nachzukommen.

Wir meinen hingegen, dass dieses Vorgehen an den Realitäten der psychiatrischen und psychotherapeutischen Versorgung vorbeigeht. Die Wirtschaftlichkeit kann mit derartigen Methoden nicht erfasst werden. Das Vorgehen produziert selbst unnötige Kosten und bindet menschliche Energien, welche anderweitig viel besser genutzt werden könnten!
Der Fachbereich der Psychiatrie und Psychotherapie ist sehr breit und umfasst viele spezifische Aufgaben und spezialisierte Tätigkeiten. Einzelne Aufgaben (Begutachtungen) können in einmaligen Sitzungen gelöst werden, andere Aufgaben (Behandlungen bei schweren psychischen Erkrankungen) erfordern oft zeitlich intensive und jahrelange Engagements. Dementsprechend sind auch die Tätigkeitsbereiche der einzelnen Psychiater sehr unterschiedlich und diese Unterschiede können nicht über einen einzigen statistischen Leisten geschlagen werden.

Den Ausgangspunkt für die Mahnbriefe bildet ein Fallkostendurchschnitt, der etwa 12 Therapiestunden pro Jahr umfasst. Dies bedeutet, dass Psychotherapeutinnen und -therapeuten, welche mit Patienten hauptsächlich in regelmässigen, längerfristigen Therapievereinbarungen mit einer Sitzungsfrequenz von 1 bis 2 Sitzungen pro Woche arbeiten, automatisch einen überdurchschnittlichen Fallkostendurchschnitt haben und so in den Verdacht der unwirtschaftlichen Arbeitsweise kommen. Wie unsinnig dies ist, sollen die folgenden Überlegungen veranschaulichen.

Nachweislich eignen sich nur bestimmte Problemstellungen für Kurzzeittherapien von 10 bis 20 Sitzungen. Kurzzeittherapien müssen zudem oft nach einer gewissen Zeit wieder aufgenommen werden oder führen dazu, dass sich die betroffenen Patienten noch tiefer mit ihren psychischen Schwierigkeiten auseinandersetzen wollen. Langzeittherapien, durchgeführt mit der Methode der psychoanalytischen Psychotherapie, haben nachweislich einen besseren, nachhaltigeren Effekt, je gründlicher und länger sie geführt werden [2]. Nachgewiesen ist auch, dass langfristige psychoanalytische Behandlungen wirtschaftlich sind, werden doch bereits nach wenigen Jahren die Ausgaben für diese Behandlung durch Vermeidung anderer medizinischer 
* WZW = Wirksamkeit $/$ Zweckmässigkeit/Wirtschaftlichkeit.

3 Breyer, Heinzel, Klein. Kosten und Nutzen ambulanter Psychoanalyse in Deutschland. Gesundheitsökonomie Qualitätsmanagement 1997;2:59-73.

4 Urteil des Eidgenössischen Versicherungsgerichtes vom 23. April 1999.

5 www.nationalegesundheit.ch.
Kosten wieder ausgeglichen [3]. Solche wissenschaftlichen Resultate legen nahe, die Wirtschaftlichkeit längerfristig zu bestimmen und sie nicht an zweifelhaften Fallkostendurchschnitten festzumachen.

Gerade im Falle der psychoanalytischen Psychotherapien ist die Problematik des Fallkostendurchschnitts schon mehrfach in den paritätischen Kommissionen verhandelt und gar bis vor Versicherungsgerichte [4] gezogen worden immer mit dem gleichen Ergebnis, dass Langzeittherapien, durchgeführt von anerkannten psychoanalytischen Psychotherapeuten, den geltenden Kriterien nach $\mathrm{WZW}^{*}$ ) genügen. Es ist daher stossend, dass die Verantwortlichen der Krankenversicherer diese Sachlage nicht zur Kenntnis nehmen wollen und nicht zusammen mit den betroffenen Organisationen nach andern Lösungen der Kontrolle der Wirtschaftlichkeit suchen.

Hochfrequente Langzeittherapien werden zumeist bei sehr schwer kranken Menschen durchgeführt. Diese Behandlungen entlasten die noch kostenaufwendigeren Psychiatrischen Kliniken und entsprechen einer Entwicklung, die aus verschiedenen Gründen eigentlich erwünscht ist. Intensive Psychotherapien werden zumeist auch bei jungen Patienten durchgeführt, um die drohende Desintegration aus dem Arbeitsprozess zu vermeiden. Auch solche Psychotherapien sind aus gesundheitspolitischer Hinsicht im Grunde erwünscht. Beide Patientengruppen (schwerkranke und junge Patienten) gehören zu den sozial eher schlechter gestellten Schichten. Beschränkungen der Leistungen können sie selbst nicht über Zusatzversicherungen auffangen; Beschränkungen würden daher den solidarischen Charakter der Krankenversicherung unterwandern.
Es ist zu befürchten, dass bei einem Wegfall der Kontrahierungsverpflichtung santésuisse nach dem Modell des Fallkostendurchschnitts ihre «vertragswürdigen» Psychiaterinnen und Psychiater und Psychotherapeutinnen und -therapeuten aussuchen würden, wie dies einzelne Kassen bei einzelnen Versicherungsplänen bereits tun. Dieses Vorgehen wäre gesundheitspolitisch aber willkürlich und kontraproduktiv. Die Folge wäre eine Destabilisierung, weil nach dem Modell des Fallkostendurchschnittes immer 20-30\% der Psychiaterinnen und Psychiater und Psychotherapeutinnen und -therapeuten «zu teuer» wären, egal wie hoch der Fallkostendurchschnitt wäre. Auf der Grundlage einer solchen Verunsicherung können keine verlässlichen therapeutischen Beziehungen aufgebaut werden, welche für die psychisch kranken Menschen aber von grösster Bedeutung sind.

Die Verbesserung der psychiatrisch-psychotherapeutischen Versorgung bildet einen Kernpunkt künftiger Gesundheitspolitik. Viele Kantone haben Psychiatriekonzepte erarbeitet, nach denen staatliche und private Anbieter aufeinander abgestimmte Versorgungsaufträge zu übernehmen haben (eine überarbeitete Gesamtplanung im Kanton Basel-Landschaft steht kurz vor der Veröffentlichung). An der Arbeitstagung Nationale Gesundheitspolitik vom 12./13. September 2002 [5] wurde der Politik für die psychische Gesundheit höchste Priorität eingeräumt und damit einmal mehr unterstrichen, dass auf diesem Gebiet ein Nachholbedarf besteht und umfassende Lösungen notwendig sind - und nicht isolierte Aktionen wie jene der santésuisse. 